\title{
Neural Network Disturbance Observer-Based Adaptive Tracking Control for Robot Manipulator
}

\author{
Hui $\mathrm{Hu}^{1}$, Peng Guo ${ }^{2}$ and Xilong $\mathrm{Qu}^{3}$ \\ ${ }^{1}$ Hunan Provincial Key Laboratory of Wind Generator and Its Control, School of \\ Electrical and Information, Hunan Institute of Engineering, Hunan Xiangtan \\ 411104, P. R. China \\ ${ }^{2,3}$ School of Computer Science and Communication, Hunan Institute of \\ Engineering, Hunan Xiangtan 411104, P. R. China \\ 21230122@qq.com,da_peng219@126.com,quxilong@126.com
}

\begin{abstract}
A novel neural network disturbance observer-based adaptive tracking control approach for robot manipulator is presented in the paper. Using the concept of 'dominant input', the robot manipulator is transferred into several SISO systems with the unknown compound disturbance which consists of external disturbance, internal parameter uncertainty and cross-coupling of subsystems. In order to reduce the restrained conditions on the disturbance, the compound disturbance is monitored by the designed neural network observer. Then parameters update laws and control laws based on Lyapunov theory and neural network observer are proposed to guarantee that all the signals in the system are uniformly ultimately bounded and achieve given tracking performance index. Finally, the simulation results are presented to demonstrate the efficiency of the control scheme.
\end{abstract}

Keywords: robot manipulator, neural network disturbance observer, tracking control, dominant input

\section{Introduction}

In the three decades, the progress of robotics goes from the standard applications of industrial robots to new fields, such as space, service robotics, medical service, and force feedback systems etc. In general, robot manipulators are multivariable nonlinear systems. Its dynamics is governed by highly nonlinear coupled, time-varying system with many uncertainties such as load variation, friction, and external disturbance etc. Due to these uncertainties system error becomes large, when a robot manipulator operates at high speed. It is a challenging problem in control field to find an effective control scheme to achieve accurate tracking of the desired motion (Khoygani et al., 2015; Singh et al., 2013; Han et al., 2015; Cuong et al., 2016).

The control technology for robotic motion and force has developed closely along with the development of control theories. Recently, the application of intelligent control such as fuzzy control and neural control to the position control of robotic manipulator have received considerable attention. A robust neural network output feedback control scheme that includes a novel neural network observer is presented for the motion control of robot manipulator (Kim et al., 1999). A kind of fuzzy disturbance observer approach is presented (Kim et al. 2002; Kim et al. 2004) for a class of SISO nonlinear system. This approach aims to observe the compound disturbance made of external disturbance and internal uncertainties through application of the universal approximation of fuzzy logic

Received (June 3, 2016), Review Result (January 13, 2017), Accepted (January 23, 2017) 
system, and to design control law of system based on this observer. A neural network disturbance observer approach for a class of MIMO system is introduced (Chen al., 2006).

To further simplify the system design, this paper proposes a novel robust tracking control method for robot manipulators with unknown external disturbances and internal uncertainties based on neural network observer. A prominent feature of this method is its application of the concept of 'dominant input' (Liu et al. 2002). That is to say, a $m \times m$ dimensional MIMO system will be decomposed into $\mathrm{m}$ SISO systems for their respective design. As for each subsystem, the neural network disturbance observer will be designed to observe the compound disturbance which consists of external disturbance, internal uncertainty and cross-coupling of subsystems, and novel control law and parameter update law will be deduced from observer to guarantee that all the signals in the system are uniformly ultimately. At the same time, robust control is introduced in controller for overcoming the effects of observational error. This paper proves the availability of the method in both theory and simulation experiment.

The rest of the paper is organized as follows. Firstly, the problem is formulated in Section II. Design of neural network disturbance observer and $\mathrm{H} \infty$ controller is given in III. Stability analysis of control system is given in Section IV. In Section V, simulation results are presented to confirm the effectiveness and applicability of the proposed method. Section VI contains the conclusions.

\section{Problem Formulation}

The traditional dynamic model of a n-Degree of Freedom (DOF) robot manipulator is presumed to be in the subsequent form by Lewis et al. (1999)

$$
H(q) \ddot{q}+C(q, \dot{q})+\phi(q)+\tau=u(t)
$$

Where $H(q) \in R^{n \times n}$ represents the inertia matrix, $C(q, \dot{q}) \in R^{n \times n}$ represents the centripetalcoriolis matrix, $\phi(q) \in R^{n}$ represents the gravity effects, $\tau \in R^{n}$ denotes the friction effects, $u(t) \in R^{n}$ signifies the torque input control vector and $q, \dot{q}, \ddot{q} \in R^{n}$ signify link position, velocity and acceleration correspondingly. Figure 1 describes the configuration of 2-DOF robot manipulator

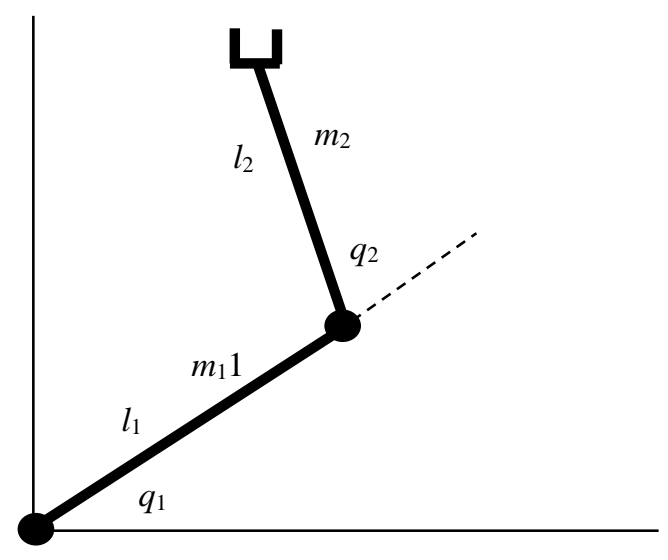

Figure 1. Configuration of 2-DOF Robot Manipulator

Where $H(q)=\left[\begin{array}{cc}\mathrm{a}_{1}+2 \mathrm{a}_{3} \cos q_{2}+2 \mathrm{a}_{4} \sin q_{2} & \mathrm{a}_{2}+\mathrm{a}_{3} \cos q_{2}+\mathrm{a}_{4} \sin q_{2} \\ \mathrm{a}_{2}+\mathrm{a}_{3} \cos q_{2}+\mathrm{a}_{4} \sin q_{2} & \mathrm{a}_{2}\end{array}\right]$, 


$$
\begin{aligned}
M(q, \dot{q})=C(q, \dot{q})+\phi(q) & =\left[\begin{array}{c}
-\left(\mathrm{a}_{3} \sin q_{2}-\mathrm{a}_{4} \cos q_{2}\right) \dot{q}_{1} \dot{q}_{2}-\left(\mathrm{a}_{3} \sin q_{2}-\mathrm{a}_{4} \cos q_{2}\right)\left(\dot{q}_{1}+\dot{q}_{2}\right) \dot{q}_{2} \\
\left(\mathrm{a}_{3} \sin q_{2}-\mathrm{a}_{4} \cos q_{2}\right) \dot{q}_{1}^{2}
\end{array}\right] \\
& =\left(\begin{array}{cc}
-h \dot{q}_{2} & -h \dot{q}_{1}-h \dot{q}_{2} \\
h \dot{q}_{1} & 0
\end{array}\right)\left[\begin{array}{c}
\dot{q}_{1} \\
\dot{q}_{2}
\end{array}\right]
\end{aligned}
$$

The other parameters are as following

$\mathrm{a}_{1}=\mathrm{I}_{1}+\mathrm{m}_{1} l_{\mathrm{cl}}^{2}+\mathrm{I}_{\mathrm{e}}+\mathrm{m}_{\mathrm{e}} l_{\mathrm{ce}}^{2}+\mathrm{m}_{\mathrm{e}} l_{1}^{2}, \mathrm{a}_{2}=\mathrm{I}_{\mathrm{e}}+\mathrm{m}_{\mathrm{e}} l_{\mathrm{ce}}^{2}, \mathrm{a}_{3}=\mathrm{m}_{\mathrm{e}} l_{1} l_{\mathrm{ce}} \cos 30^{\circ}, \mathrm{a}_{4}=\mathrm{m}_{\mathrm{e}} l_{1} l_{\mathrm{ce}} \sin 30^{\circ}, \mathrm{m}_{1}=1 \mathrm{~kg}$ $, \mathrm{m}_{\mathrm{e}}=2 \mathrm{~kg}, l_{1}=1 \mathrm{~m}, l_{\mathrm{c} 1}=0.5 \mathrm{~m}, l_{\mathrm{ce}}=0.6 \mathrm{~m}, \mathrm{I}_{1}=0.12 \mathrm{kgm}^{2}, \mathrm{I}_{\mathrm{e}}=0.25 \mathrm{kgm}^{2}$.

According to the above parameters, $H(q)=\left[\begin{array}{ll}H_{11} & H_{12} \\ H_{21} & H_{22}\end{array}\right]$ is a positive definite matrix. Rewriting equation (1) as

$$
\begin{aligned}
{\left[\begin{array}{l}
\ddot{q}_{1} \\
\ddot{q}_{2}
\end{array}\right] } & =\left[\begin{array}{ll}
H_{11} & H_{12} \\
H_{21} & H_{22}
\end{array}\right]^{-1}\left(-\left(\begin{array}{cc}
-h \dot{q}_{2} & -h \dot{q}_{1}-h \dot{q}_{2} \\
h \dot{q}_{1} & 0
\end{array}\right)\left[\begin{array}{l}
\dot{q}_{1} \\
\dot{q}_{2}
\end{array}\right]-\left[\begin{array}{c}
\tau_{1} \\
\tau_{2}
\end{array}\right]+\left[\begin{array}{l}
u_{1} \\
u_{2}
\end{array}\right]\right) \\
& =\frac{1}{H_{11} H_{22}-H_{12} H_{21}}\left\{\left(\begin{array}{ll}
-H_{22} h \dot{q}_{2}+H_{12} h \dot{q}_{1} & H_{22}\left(h \dot{q}_{1}+h \dot{q}_{2}\right) \\
-H_{21} h \dot{q}_{2}-H_{11} h \dot{q}_{1} & -H_{21}\left(h \dot{q}_{1}+h \dot{q}_{2}\right)
\end{array}\right)\left[\begin{array}{c}
\dot{q}_{1} \\
\dot{q}_{2}
\end{array}\right]+\left(\begin{array}{cc}
H_{22} & -H_{12} \\
-H_{21} & H_{11}
\end{array}\right)\left[\begin{array}{l}
u_{1} \\
u_{2}
\end{array}\right]\right\}
\end{aligned}
$$

The relative order of the system is $\left[r_{1}, r_{2}\right]=[2,2]$.Defining $x^{T}=\left[x_{1}^{T}, x_{2}^{T}\right], x_{i}^{T}=\left[q_{i}, \dot{q}_{i}\right]$, $i=1,2$.

Rewriting equation (2) as

$$
\left\{\begin{array}{l}
\dot{x}=A^{\prime} x+B^{\prime}[F(x)+G(x) u+\tau(x)] \\
y=C^{\prime T} x
\end{array}\right.
$$

According to the relative order of the system, the input-output equation is

$$
\left[\begin{array}{l}
y_{1}^{\left(r_{1}\right)} \\
y_{2}^{\left(r_{2}\right)}
\end{array}\right]=F(x)+\Delta F(x)+G(x) u+\Delta G(x) u+\tau(x), y_{1}=x_{11}, y_{2}=x_{21}
$$

Where

$$
\begin{gathered}
F(x)=\left[\begin{array}{l}
f_{1}(x) \\
f_{2}(x)
\end{array}\right]=-H^{-1}(q) M(q, \dot{q}), G(x)=\left[\begin{array}{ll}
g_{11}(x) & g_{12}(x) \\
g_{21}(x) & g_{22}(x)
\end{array}\right]=H^{-1}(q) . \Delta F(x)=\left[\begin{array}{c}
\Delta f_{1}(x) \\
\Delta f_{2}(x)
\end{array}\right] \text { and } \\
\Delta G(x)=\left[\begin{array}{ll}
\Delta g_{11}(x) & \Delta g_{12}(x) \\
\Delta g_{21}(x) & \Delta g_{22}(x)
\end{array}\right] \text { are the parameter uncertainty. } \\
\tau(q, \dot{q})=\left[\begin{array}{l}
\tau_{1}(q, \dot{q}) \\
\tau_{2}(q, \dot{q})
\end{array}\right]=-H^{-1}(q) \tau, A^{\prime}=\operatorname{diag}\left[A_{1}^{\prime}, A_{2}^{\prime}\right], B^{\prime}=\operatorname{diag}\left[B_{1}^{\prime}, B_{2}^{\prime}\right], C^{\prime T}=\operatorname{diag}\left[C_{1}^{\prime}, C_{2}^{\prime}\right] \\
A_{i}^{\prime}=\left[\begin{array}{ll}
0 & 1 \\
0 & 0
\end{array}\right], B_{i}^{\prime}=\left[\begin{array}{l}
0 \\
1
\end{array}\right], C_{i}^{\prime}=\left[\begin{array}{ll}
1 & 0
\end{array}\right], i=1,2 .
\end{gathered}
$$

The $2 \times 2$-dimensional MIMO system given in (4) can be seen as 2 SISO systems. And its subsystem is as the following

$$
y_{i}^{\left(r_{i}\right)}=f_{i}(x)+\Delta f_{i}(x)+g_{i 1}(x) u_{1}+g_{i 2}(x) u_{2}+\sum_{j=1}^{2} \Delta g_{i j}(x) u_{j}+\tau_{i}
$$

and $i=1,2$. The output of this subsystem will be influenced by the input from $u_{1}$ to $u_{2}$.

Choosing an input as 'dominant input' among these 2 inputs marked as ${ }^{u_{i}}$ and the rest will be taken as the disturbance. We obtain

$$
\begin{aligned}
& y_{i}^{\left(r_{i}\right)}=f_{i}(x)+g_{i i}(x) u_{i}+\omega_{i}(x, u) \\
& \omega_{i}(x, u)=\Delta f_{i}(x)+\sum_{j=1}^{2} \Delta g_{i j}(x) u_{j}+\sum_{\substack{j=1 \\
j \neq i}}^{2} g_{i j}(x) u_{j}+\tau_{i}
\end{aligned}
$$

In this way, a $2 \times 2$-dimensional MIMO system can be decomposed into 2 SISO systems. 
In this paper, the universal approximation of neural networks is used as disturbance observer to observe the overall compound disturbance of system. And a robust adaptive controller is designed consequently to make input $y_{i}$ in system (3) and $y_{i}, \cdots, y_{i}^{\left(r_{i}-1\right)}$ to track the bounded reference signal $y_{i d}$ and its corresponding derivatives. As for a given disturbance attenuation level constant $\rho>0$, the following $\mathrm{H} \infty$ tracking performance index will be obtained

$$
\frac{1}{2} \int_{0}^{T} e^{T} Q e d t \leq \frac{1}{2} e^{T}(0) P e(0)+\sum_{i=1}^{2} \frac{1}{2 \gamma_{i}} \tilde{\theta}_{i}^{T}(0) \tilde{\theta}_{i}(0)+\sum_{i=1}^{2}\left(\frac{1}{2} \varsigma_{i}^{2}(0)+\int_{0}^{T}\left(\frac{1}{2} \rho^{2}+\frac{1}{2 \sigma_{i}}\right) \bar{\varepsilon}_{i}^{2} d t\right)
$$

Where $\gamma_{i}>0$ is learning rate. $\sigma_{i}>0$ is design parameter, $T \in[0, \infty), e=\left[e_{1}^{T}, e_{2}^{T}\right]^{T}$ are output vectors of tracking error. $\tilde{\theta}_{i}$ is vector of parameter approximating error.$\varsigma_{i}$ is error of disturbance observation. $\bar{\varepsilon}_{i}$ is the upper bound for neural networks approximating error. $P=\operatorname{diag}\left(P_{1}, P_{2}\right), Q=\operatorname{diag}\left(Q_{1}, Q_{2}\right)$.

\section{Designs of Neural Network Disturbance Observer and Ho Controller}

Considering system (6), and we also define

$$
\bar{e}_{i}=y_{i d}-y_{i}, e_{i}=\left[\bar{e}_{i}, \dot{\bar{e}}_{i} \ldots \bar{e}_{i}^{\left(r_{i}-1\right)}\right]^{T}, k_{i}=\left[k_{i i_{i}} \cdots k_{i i_{1}}\right]^{T} \in R^{r_{i}}
$$

The output of neural network disturbance observer is $\hat{\omega}_{i}$. If $\hat{\omega}_{i}$ can approximate the disturbance $\omega_{i}$ of system well, then we can choose the controller in the following form for system (6) with considering $g_{i i}(x) \neq 0$

$$
u_{i}=\frac{1}{g_{i i}(x)}\left(-f_{i}(x)+v_{i}-\hat{\omega}_{i}-u_{i a}\right)
$$

where $v_{i}=y_{i d}^{\left(r_{i}\right)}+k_{i}^{T} e_{i}, u_{i a}$ is the robust control input proposed subsequently.

Substituting (8) to (6), we can get

$$
e_{i}^{\left(r_{i}\right)}=-k_{i}^{T} e_{i}+\hat{\omega}_{i}-\omega_{i}+u_{i a}
$$

Rewriting equation (9) as following

$$
\dot{e}_{i}=A_{i} e_{i}+B_{i}\left(\hat{\omega}_{i}-\omega_{i}\right)+B_{i} u_{i a}
$$

where $A_{i}=\left[\begin{array}{ccccc}0 & 1 & 0 & \cdots & 0 \\ 0 & 0 & 1 & \cdots & 0 \\ \vdots & \vdots & \vdots & \vdots & \vdots \\ -k_{i r_{i}} & -k_{i_{r_{-1}}} & -k_{i_{r_{-2}}} & \cdots & -k_{i r_{1}}\end{array}\right], B_{i}=\left[\begin{array}{c}0 \\ 0 \\ \vdots \\ 1\end{array}\right]$.

Assumption 1: if $x \in M_{x}, M_{x}$ is a compact set, then the optimal parameter of neural networks is defined as

$$
\theta_{i}^{*}=\underset{\theta_{i} \in \Omega_{i}}{\arg \min }\left[\sup _{x \in M_{x}}\left|\omega_{i}-\hat{\omega}_{i}\right|\right]
$$

where $\Omega_{i}=\left\{\theta_{i}\left\|\theta_{i}\right\| \leq M_{i}\right\}$ means the subset with hope border, $M_{i}$ is the design parameter. And the output of neural network disturbance observer is $\hat{\omega}_{i}=\theta_{i}^{T} \xi . \theta_{i}=\left(\theta_{i 1}, \cdots, \theta_{i M}\right)^{T}$ is adjustable parameter vector. $\xi(x)=\left(\xi_{1}(x) \cdots \xi_{M}(x)\right)^{T}$ is the basis function vector.

According to universal approximation theorem, the existence of optimal parameter $\theta_{i}^{*}$ can make $\hat{\omega}_{i}\left(x \mid \theta_{i}^{*}\right)$ approximate to compound disturbance $\omega_{i}$ as much as possible. Therefore

$$
\omega_{i}(x)=\hat{\omega}_{i}\left(x \mid \theta_{i}^{*}\right)+\varepsilon_{i}=\theta_{i}^{* T} \xi+\varepsilon_{i},\left\|\varepsilon_{i}\right\| \leq \bar{\varepsilon}_{i}
$$

where $\varepsilon_{i}$ is the approximation error. $\bar{\varepsilon}_{i}$ is the upper bound of approximation error. Then equation (10) can be written as 


$$
\begin{aligned}
\dot{e}_{i} & =A_{i} e_{i}+B_{i}\left(\theta_{i}^{T} \xi(x)-\theta_{i}^{* T} \xi(x)-\varepsilon_{i}\right)+B_{i} u_{i a} \\
& =A_{i} e_{i}+B_{i} \tilde{\theta}_{i}^{T} \xi(x)+B_{i} u_{i a}-B_{i} \varepsilon_{i}
\end{aligned}
$$

$\tilde{\theta}_{i}=\theta_{i}-\theta_{i}^{*}$ is the parameter approximating error. The Ho controller of error is chosen as

$$
u_{i a}=-\frac{1}{r} B_{i}^{T} P_{i} e_{i}
$$

$P_{i}=P_{i}^{T} \geq 0$ is the solution to the following equation

$$
P_{i} A_{i}+A_{i}^{T} P_{i}+Q_{i}-\frac{2}{r} P_{i} B_{i} B_{i}^{T} P_{i}+\frac{1}{\rho^{2}} P_{i} B_{i} B_{i}^{T} P_{i}=0
$$

Where $Q_{i}>0$. The proper weight is chosen to make $2 \rho^{2} \geq r$ to obtain a symmetric positive semi-definite solution. Choosing $i$-th disturbance observer system as the following

$$
\begin{aligned}
& \dot{\mu}_{i}=-\sigma_{i} \mu_{i}+p_{i}\left(x, \hat{\omega}_{i}\right), \quad \mu_{i} \in R^{p} \\
& \text { where } p_{i}\left(x, \hat{\omega}_{i}\right)=\sigma_{i} y_{i}^{\left(r_{i}-1\right)}+f_{i}(x)+g_{i i}(x) u+\hat{\omega}_{i} .
\end{aligned}
$$

Define disturbance observation error $\varsigma_{i}=y_{i}^{\left(r_{i}-1\right)}-\mu_{i}$, then from (6) and (16), the dynamic of disturbance observation error can be derived as

$$
\dot{\zeta}_{i}=-\sigma_{i} \varsigma_{i}+\omega_{i}-\hat{\omega}_{i}
$$

If $\varsigma_{i} \rightarrow 0$, which means $\mu_{i} \rightarrow y_{i}^{\left(r_{i}-1\right)}$, then output $\hat{\omega}_{i}$ of neural network is proved to approximate to unknown disturbance $\omega_{i}$.

Theorem 1: Assuming using neural network disturbance observer (16) to estimate disturbance, taking the controller (8) as the controller of system (6), and choosing adaptive law of neural network parameters as:

$$
\dot{\theta}_{i}=\gamma_{i} e_{i}^{T} P_{i} B_{i} \xi(x)+\gamma_{i} \varsigma_{i} \xi(x)
$$

Then the observation errors are uniformly ultimately bounded, and the tracking error of system (6) is smaller than the given performance index:

$$
\frac{1}{2} \int_{0}^{T} e_{i}^{T} Q_{i} e_{i} d t \leq \frac{1}{2} e_{i}^{T}(0) P_{i} e_{i}(0)+\frac{1}{2 \gamma_{i}} \tilde{\theta}_{i}^{T}(0) \tilde{\theta}_{i}(0)+\frac{1}{2} \varsigma_{i}^{2}(0)+\int_{0}^{T}\left(\frac{1}{2} \rho^{2}+\frac{1}{2 \sigma_{i}}\right) \bar{\varepsilon}_{i}^{2} d t
$$

$\gamma_{i}>0$ is learning rate. $\sigma_{i}>0$ is design parameter. $\tilde{\theta}_{i}=\theta_{i}^{*}-\theta_{i}$.

Proof: Choosing an adequate Lyapunov function as

$$
V_{i}=\frac{1}{2} e_{i}^{T} P_{i} e_{i}+\frac{1}{2 \gamma_{i}} \tilde{\theta}_{i}^{T} \tilde{\theta}_{i}+\frac{1}{2} \varsigma_{i}^{2}
$$

Taking the time derivative of $V_{i}$, and considering (14), then we get

$$
\dot{V}_{i}=\frac{1}{2} e_{i}^{T}\left(A_{i}^{T} P_{i}+P_{i} A_{i}\right) e_{i}+e_{i}^{T} P_{i} B_{i} \tilde{\theta}_{i}^{T} \xi(x)+e_{i}^{T} P_{i} B_{i} u_{i a}-e_{i}^{T} P_{i} B_{i} \varepsilon_{i}+\frac{1}{\gamma_{i}} \dot{\tilde{\theta}}_{i}^{T} \tilde{\theta}_{i}+\varsigma_{i} \dot{s}_{i}
$$

Taking (14) , (15) ,（17），(18） into account in above equation.

$$
\dot{V}_{i}=-\frac{1}{2} e_{i}^{T} Q_{i} e_{i}-\frac{1}{2 \rho^{2}} e_{i}^{T} P_{i} B_{i} B_{i}^{T} P_{i} e_{i}-e_{i}^{T} P_{i} B_{i} \varepsilon_{i}-\sigma_{i} \varsigma_{i}^{2}+\varsigma_{i} \bar{\varepsilon}_{i}
$$

We know $-\sigma_{i} \varsigma_{i}^{2}+\varsigma_{i} \bar{\varepsilon}_{i} \leq-\frac{\sigma_{i}}{2} \varsigma_{i}^{2}+\frac{1}{2 \sigma_{i}} \bar{\varepsilon}_{i}^{2}$, then

$$
\begin{aligned}
& \dot{V}_{i} \leq-\frac{1}{2} e_{i}^{T} Q_{i} e_{i}-\frac{1}{2 \rho^{2}} e_{i}^{T} P_{i} B_{i} B_{i}^{T} P_{i} e_{i}-e_{i}^{T} P_{i} B_{i} \varepsilon_{i}+\frac{1}{2 \sigma_{i}} \bar{\varepsilon}_{i}^{2} \\
& =-\frac{1}{2} e_{i}^{T} Q_{i} e_{i}-\frac{1}{2}\left[\left(\frac{1}{\rho} B_{i}^{T} P_{i} e_{i}+\rho \varepsilon_{i}\right)^{T}\left(\frac{1}{\rho} B_{i}^{T} P_{i} e_{i}+\rho \varepsilon_{i}\right)\right]
\end{aligned}
$$




$$
\begin{aligned}
&+\frac{1}{2} \rho^{2} \varepsilon_{i}^{2}+\frac{1}{2 \sigma_{i}} \bar{\varepsilon}_{i}^{2} \\
& \leq-\frac{1}{2} \lambda_{\min }\left(Q_{i}\right)\left\|e_{i}\right\|^{2}+\frac{1}{2} \rho^{2} \bar{\varepsilon}_{i}^{2}+\frac{1}{2 \sigma_{i}} \bar{\varepsilon}_{i}^{2} \\
& \text { If }\left\|e_{i}\right\| \geq \sqrt{\frac{1}{\lambda_{\min }\left(Q_{i}\right)}}|\rho| \bar{\varepsilon}_{i}+\sqrt{\frac{1}{\sigma_{i} \lambda_{\min }\left(Q_{i}\right)}} \bar{\varepsilon}_{i} \text { then } \dot{V}_{i}<0 .
\end{aligned}
$$

The Lyapunov function for the overall system structure is $V=V_{1}+V_{2}$, taking the time derivative of $V$,we get $\dot{V}=\dot{V}_{1}+\dot{V}_{2}$. Then

$$
\dot{V}=\dot{V}_{1}+\dot{V}_{2}<0
$$

So, the closed-loop system's stability is guaranteed with the effect of designed controller. And the error of observer will be uniformly ultimately bounded.

Integrating equation (24), we can get

$$
V_{i}(T)-V_{i}(0) \leq-\frac{1}{2} \int_{0}^{T} e_{i}^{T} Q_{i} e_{i} d t+\int_{0}^{T}\left(\frac{1}{2} \rho^{2} \bar{\varepsilon}_{i}^{2}+\frac{1}{2 \sigma_{i}} \bar{\varepsilon}_{i}^{2}\right) d t
$$

Because $V_{i}(T) \geq 0$, according to (25),we can get equation (19). This completes the proof. The overall system will get $\mathrm{H} \infty$ tracking performance (7).

\section{Simulation Study}

The design parameters are selected as

$$
Q_{1}=Q_{2}=\left(\begin{array}{cc}
10 & \\
& 10
\end{array}\right), \quad \rho=0.05, \quad r=0.005, \quad k_{1}=k_{2}=[1,2]^{T}, \quad \sigma_{1}=\sigma_{2}=10,
$$

$\gamma_{1}=18, \gamma_{2}=5 . P_{1}=P_{2}=\left(\begin{array}{cc}15 & 5 \\ 5 & 5\end{array}\right)$ is the solution to Riccati equation (15). The control objective is to force the system output $y$ to follow the desired trajectory that is employed as $q_{d}=\left[q_{1 d}, q_{2 d}\right]=\left[\cos \frac{\pi}{4}(\mathrm{t}-0.5), \cos \left(\frac{\pi}{6} t\right)\right]$.

RBF neural network disturbance observer is $\hat{\omega}_{i}=\theta_{i}^{T} \xi, i=1,2 . \xi(x)=\left(\xi_{1}(x) \cdots \xi_{M}(x)\right)^{T}$ is the basic function vector, where $\xi_{i}(x)=\exp \left[\frac{-\left(x-\mu_{i}\right)^{T}\left(x-\mu_{i}\right)}{\sigma_{i}^{2}}\right], i=1, \cdots, M$, $\mu_{i}=\left[\mu_{i 1}, \mu_{i 2}, \mu_{i 3}, \mu_{i 4}\right]^{T}$ is the center of Gaussian function. $\sigma_{i}$ is the width. $M$ is the node number.

According to the design process, we can get the control law as following

$$
\begin{aligned}
& u_{1}=\frac{1}{g_{11}(q)}\left(-f_{1}(q, \dot{q})+\ddot{q}_{1 d}+e_{1}+2 \dot{e}_{1}-\theta_{1}^{T} \xi(x)-u_{1 a}\right) \\
& u_{1 a}=-200\left(5 e_{1}+5 \dot{e}_{1}\right) \\
& \dot{\theta}_{1}=18\left(5 e_{1}+5 \dot{e}_{1}\right) \xi(x)+18 \varsigma_{1} \xi(x) \\
& \dot{\varsigma}_{1}=-10 \varsigma_{1}-\tilde{\theta}_{1} \xi(x) \\
& u_{2}=\frac{1}{g_{22}(q)}\left(-f_{2}(q, \dot{q})+\ddot{q}_{2 d}+e_{2}+2 \dot{e}_{2}-\theta_{2}^{T} \xi(x)-u_{2 a}\right) \\
& u_{2 a}=-200\left(5 e_{2}+5 \dot{e}_{2}\right) \\
& \dot{\theta}_{2}=5\left(5 e_{2}+5 \dot{e}_{2}\right) \xi(x)+5 \varsigma_{2} \xi(x) \\
& \dot{\varsigma}_{2}=-10 \varsigma_{2}-\tilde{\theta}_{2} \xi(x)
\end{aligned}
$$


The system initial conditions are $x(0)=[1,0,0,0]^{T}$.Considering the disturbance $\tau=[0.1 \sin (0.3 t), 0.2 \sin (0.2 t)]$, The simulation results using MATLAB are shown in Figure 2 and Figure 3.

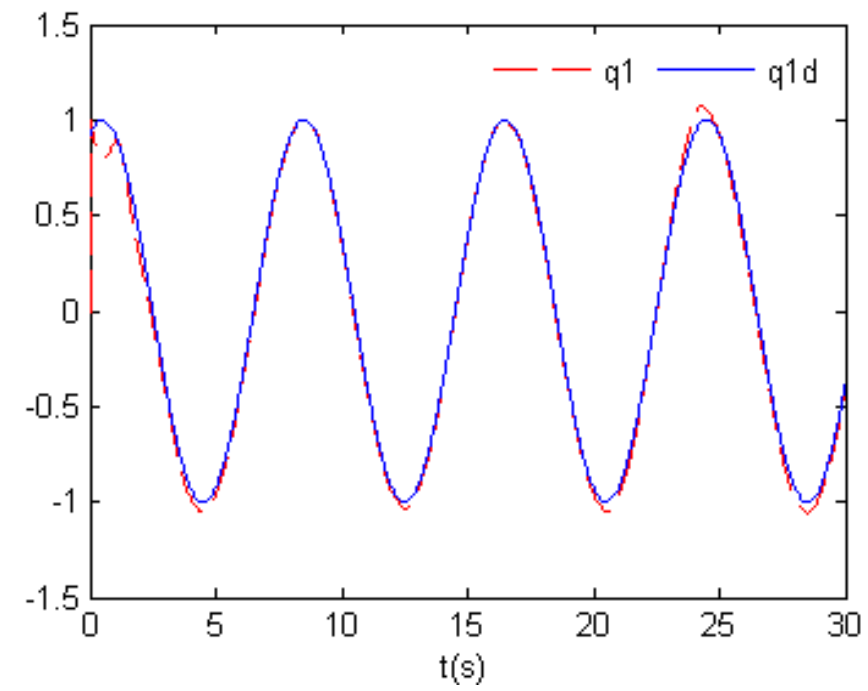

Figure 2. Plots of Output 1Tracking of System

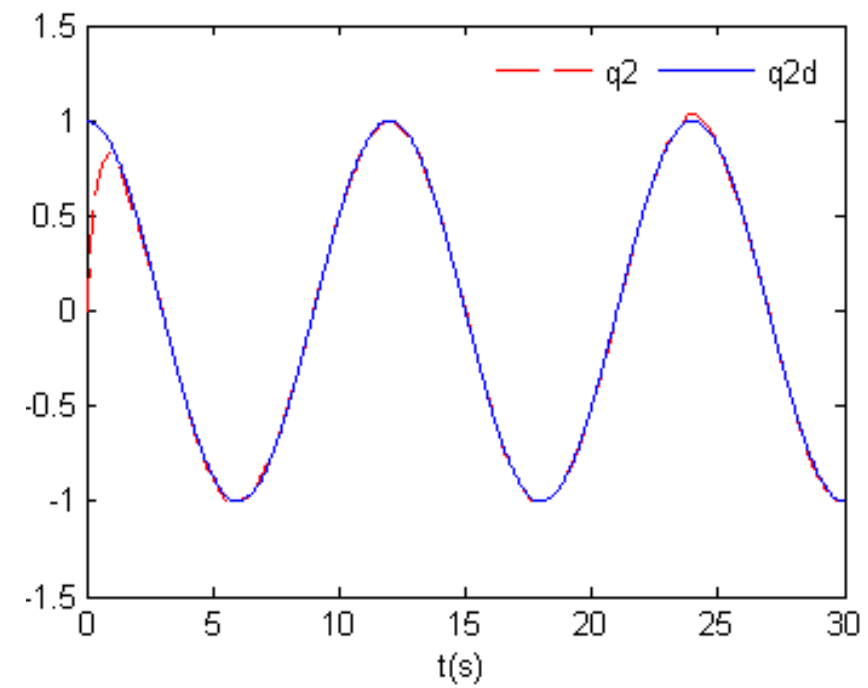

Figure 3. Plots of Output 2Tracking of System

Considering the influence of system parameters uncertainty, $\Delta F(q, \dot{q})=\left[\begin{array}{c}-0.25 \sin \left(q_{1}\right) \\ 0.25 \sin \left(q_{2}\right)\end{array}\right]$,
$\Delta G(q, \dot{q})=\left[\begin{array}{cc}0.25 & 0.125\left(\sin \left(q_{1}\right) \sin \left(q_{2}\right)+\cos \left(q_{1}\right) \cos \left(q_{2}\right)\right) \\ -0.125\left(\sin \left(q_{1}\right) \sin \left(q_{2}\right)+\cos \left(q_{1}\right) \cos \left(q_{2}\right)\right) & 0.25\end{array}\right]$

The simulation results are shown in Figure 4 and Figure 5. 


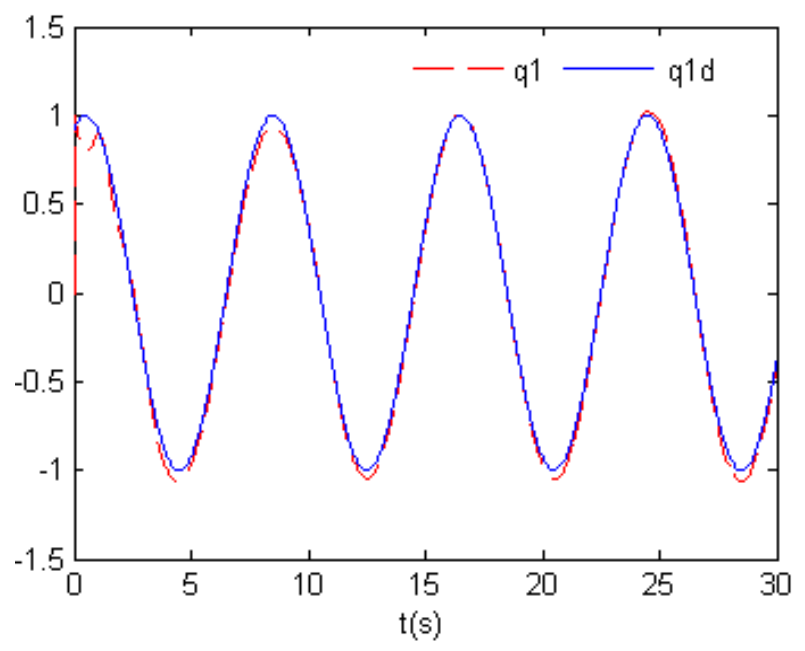

Figure 4. Plots of Output 1Tracking of System

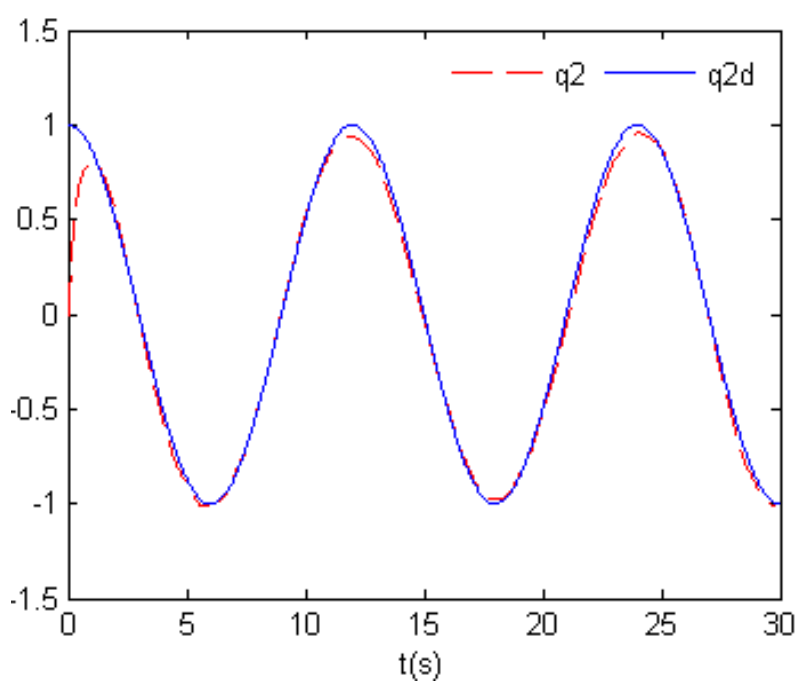

Figure 5. Plots of Output 2Tracking of System

It can be seen that the actual trajectory converges rapidly to the desired one and the system has strong anti-disturbance ability. It can also realize the tracking control when the system has the parameters uncertainty and disturbance. These simulation results demonstrate the tracking capability of the proposed controller and its effectiveness for control tracking of robot manipulator with disturbance and parameter uncertainty.

\section{Conclusions}

The paper presents a neural network tracking control approach based on disturbance observer for robot manipulator with unknown external disturbance and parameter uncertainty. By using the concept of 'dominant input', the robot manipulator is transferred into several SISO systems with the unknown compound disturbance which consists of external disturbance, internal parameter uncertainty and cross-coupling of subsystems. The compound disturbance is monitored by the designed neural network observer and then control law and parameters update law of system are designed based on the neural network disturbance observer. The simulation results show that the tracking capability of the proposed controller which can guarantee all the signals in the system are uniformly ultimately bounded. Our further research will be focused on such applications of the proposed neural network disturbance observer. 


\section{Acknowledgment}

It is a project supported by National Natural Science Fund, China (Grant No.5177040). Supported by Scientific Research Fund of Hunan Provincial Education Department, China (Study of Intelligent Vehicle's Lateral Robust Stable Controller on Neural Network). Supported by Provincial Natural Science Foundation of Hunan, China (Grant No. 13JJ9022). Supported by Provincial Natural Science Foundation of Hunan, China (Grant No. 14JJ6041). Supported by the Construct Program of the Key Discipline in Hunan Province: Control Science and Engineering, Science and Technology Innovation Team of Hunan Province: Complex Network Control. Supported by CIC-WEP.

\section{References}

[1] M.R.R. Khoygani and A.R. Vali, "Intelligent nonlinear observer design for a class of nonlinear discretetime flexible joint robot", Intel Serv Robotics, no. 8, (2015), pp. 45-56.

[2] H.P. Singh and N. Sukavanam, "Stability analysis of robust adaptive hybrid position/force controller for robot manipulators using neural network with uncertainties", Neural Computer \& Application, no. 22, (2013), pp. 1745-1755.

[3] S. Han and J.M Lee, "Decentralized neural network control for guaranteed tracking error constraint of a robot manipulator", Internal Journal of Control, Automation and Systems, vol. 13, no. 4, (2015), pp. 906-915.

[4] P.V. Cuong and Y.N. Wang, "Adaptive trajectory tracking neural network control with robust compensator for robot manipulators", Neural Computer \&Application, no. 27, (2016), pp. 525-536.

[5] W. RJ and C. PC, "Robust neural-fuzzy-network control for robot manipulator including actuator dynamics", IEEE Trans Ind Electron, vol. 53, no. 4, (2006), pp. 1328-1349.

[6] Z. Song, J. Yi, D. Zhao, X. Li, "A computed torque controller for uncertain robotic manipulator systems: fuzzy approach", Fuzzy Sets Systems, no. 154, (2005), pp. 208-216.

[7] Y.H Kim, F.L Lewis, "Neural network output feedback control of robot manipulators", IEEE Transactions on Robot Automation, vol. 15, no. 2, (1999), pp. 301-309.

[8] E. Kim, "A fuzzy disturbance observer and its application to control", IEEE Transactions on fuzzy systems, vol. 10, no. 1, (2002), pp. 77-84.

[9] E. Kim and C. Park, "Fuzzy disturbance observer approach to robust tracking control of nonlinear sampled systems with the guaranteed suboptimal Ho performance", IEEE Transactions on systems, man, and cybernetics, vol. 34, no.3, (2004), pp. 1574-1581

[10] C. Mou, J. Iang, C. Sheng and W. Qing-Xian, "Robust H-infinity control of a class of nonlinear uncertain systems with disturbance observer", Control Theory\&Applications, vol. 23, no. 4, (2006), pp. 611-614.

[11] L. Guo-rong and W. Bai-wu, "Direct adaptive fuzy robust control for a class of nonlinear MIMO systems", Control Theory\&Applications, vol. 19, no. 5, (2002), pp. 693-698

[12] F.L. Lewis, S. Jagannathan and A. Yesildirek, "Neural network control of Robot Manipulators and NonLinear Systems", 1st ed.UK:Taylor\&Francis, (1999).

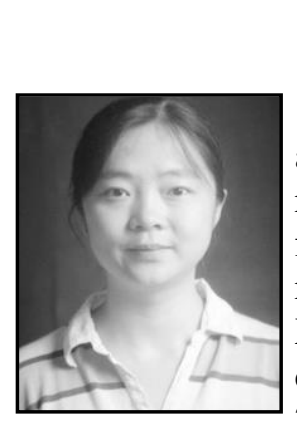

\section{Authors}

Hui Hu, she is an associate professorr of Department of electrical and information engineering, Hunan institute of engineering. Dr. Hu received the B.S. degree in electronics and information engineering from Hunan University of Science and Technology in 2001.And received the M.S. degree in power electronics and drives from Xiangtan University in 2004. And received the $\mathrm{Ph}$. D degree in control theory and control engineering from Hunan University in 2010. Her research interests include nonlinear systems tracking control, MIMO systems control, uncertain nonlinear system contorl and intelligent control. 


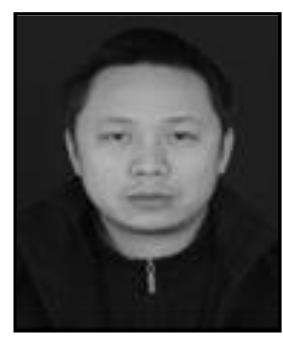

Peng Guo, he is an associate professor of Department of Computer and Science, Hunan institute of engineering. He received the B.S. degree in electronics and information engineering from Hunan University of Science and Technology in 2000 and received the M.S. degree in computer science from Hunan University in 2006. His research interests include intelligent control and computing theory, multimedia computing and networking and agent technology.

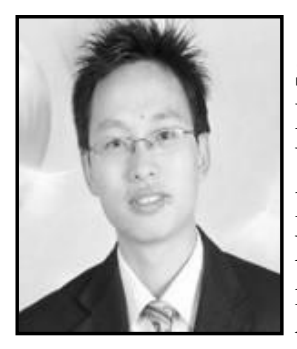

Xilong Qu, he was born in 1978. He received his $\mathrm{Ph}$. D from Southwest Jiaotong Universityin 2006. He is an associate professor of Hunan Institute of Engineering, master supervisor of Xiangtan University, the key young teacher of Hunan province, academic leader of computer application technology in Hunan Institute of Engineering. His research interesting is web service technology, information safety and networked manufacturing. He presided the projects supported by ScientificResearch Funoof Hunan Provincial Education Department (08A009), the projects supported by Provincial Natural Science Foundation of Hunan (13JJ9022) and the project supported by Provincial Science \& Technology plan project of Hunan (2010GK3048). He has published more than 30 papers in some important magazines. 